\title{
Profiling the transcriptome of Gracilaria changii (Rhodophyta) in response to light deprivation.
}

\begin{abstract}
Light regulates photosynthesis, growth and reproduction, yield and properties of phycocolloids, and starch contents in seaweeds. Despite its importance as an environmental cue that regulates many developmental, physiological, and biochemical processes, the network of genes involved during light deprivation are obscure. In this study, we profiled the transcriptome of Gracilaria changii at two different irradiance levels using a cDNA microarray containing more than 3,000 cDNA probes. Microarray analysis revealed that 93 and 105 genes were up- and down-regulated more than 3-fold under light deprivation, respectively. However, only $50 \%$ of the transcripts have significant matches to the nonredundant peptide sequences in the database. The transcripts that accumulated under light deprivation include vanadium chloroperoxidase, thioredoxin, ferredoxin component, and reduced nicotinamide adenine dinucleotide dehydrogenase. Among the genes that were down-regulated under light deprivation were genes encoding light harvesting protein, light harvesting complex I, phycobilisome $7.8 \mathrm{kDa}$ linker polypeptide, low molecular weight early light-inducible protein, and vanadium bromoperoxidase. Our findings also provided important clues to the functions of many unknown sequences that could not be annotated using sequence comparison.
\end{abstract}

Keyword: cDNA microarray; Irradiance; Gracilaria changii; Light deprivation; Seaweed. 